\title{
El control de la presión arterial y su influencia en el estilo de vida de las personas hipertensas
}

\section{The control of blood pressure and its influence on the lifestyle of hypertensive people}

El control de la presión arterial

Reynier García Rodríguez ${ }^{(1)}$

Leyra Adriana Fonseca Liermo ${ }^{(2)}$

Francisco Dueñas Segovia ${ }^{(3)}$

Lenin Mauricio Andaluz Granda ${ }^{(4)}$

(1) Instituto Superior Tecnológico Portoviejo. Ecuador. email: reygarod79@gmail.com ORCID: https://orcid.org/0000-0002-4160-5749

(2) Universidad de Ciencias Médicas de La Habana. Cuba. email: leyadriana99@gmail.com

(3) Instituto Superior Tecnológico Portoviejo. Ecuador. email: francisco.duenas20@,hotmail.com

(4) Instituto Superior Tecnológico Portoviejo. Ecuador. email: mandaluz1987@,hotmail.com

Contacto: reygarod79@gmail.com

\section{Recibido: 1-11-2020 Aprobado: 15-12-2020}

\section{Resumen}

A nivel internacional la hipertensión arterial es una problemática acuciante y en Ecuador también, por tal razón el artículo se gesta ante el carente control de la hipertensión arterial en usuarios y la necesidad del logro de estilos de vida saludables que sean de impacto en las personas hipertensas y en la comunidad, en sentido general. La investigación forma parte del proyecto de investigación, "Zona protegida, Parques La Rotonda y Las Vegas de Portoviejo”, como iniciativa del Instituto Superior Tecnológico "Portoviejo". El objetivo de estudio fue, sensibilizar a las personas hipertensas en el control de la presión arterial en función de que puedan lograr un estilo de vida saludable. El estudio 
fue descriptivo de corte transversal, realizado durante el semestre junio-diciembre de 2019, con una muestra de 500 personas comprendidas entre 30 y 80 años de edad, todos dieron el consentimiento informado. Los principales resultados destacan que se logró identificar los principales factores por los cuales la población estudiada padece de hipertensión arterial y se revela sus estilos de vida que van en detrimento de la salud integral, pero con énfasis en la hipertensión arterial como problema de salud comunitario. Se concluye que la hipertensión arterial es multicausal, ha sido estudiada por diferentes especialistas del área médica y otras apelándose a que su control radica en los buenos hábitos relacionados con la alimentación, la nutrición, el ejercicio físico, la reducción del estrés y la ansiedad; propendiendo a una comunidad poseedora de una vida saludable.

Palabras clave: presión arterial, persona hipertensa, estilo de vida

\begin{abstract}
At an international level, arterial hypertension is a pressing problem and in Ecuador as well, for this reason the article is gestated in light of the lack of control of arterial hypertension in users and the need to achieve healthy lifestyles that have an impact on hypertensive people and in the community, in a general sense. The research is part of the research project, "Protected Area, La Rotonda and Las Vegas de Portoviejo Parks", as an initiative of the "Portoviejo" Higher Technological Institute. The objective of the study was to sensitize hypertensive people in the control of blood pressure in order to achieve a healthy lifestyle. The study was descriptive, cross-sectional, carried out during the June-December 2019 semester, with a sample of 500 people between 30 and 80 years of age, all of whom gave informed consent. The main results highlight that it was possible to identify the main factors by which the studied population suffers from arterial hypertension and reveals their lifestyles that are detrimental to comprehensive health, but with emphasis on arterial hypertension as a community health problem. It is concluded that arterial hypertension is multicausal, it has been studied by different specialists in the medical area and others appealing that its control lies in good habits related to food, nutrition, physical exercise, the reduction of stress and anxiety; tending to a community with a healthy life.
\end{abstract}

Keywords: blood pressure, hypertensive person, lifestyle 


\section{Introducción}

La sociedad tiene ante sí el reto permanente de cuidar su salud, lo que se logra a través de la prevención o profilaxis. La Organización Mundial de la Salud (OMS, 2019) planteó que: "la salud es satisfacción plena en los aspectos físico, psicológico, mental y social; no limitándose a la ausencia de alguna patología y afección de diversa índole" (OMS, 2019). Sobre esta base, cada persona debe reflexionar en torno a la salud integral con énfasis en los factores que influyen en la calidad de vida, la felicidad de la persona y el disfrute plena de su salud.

En la actualidad se ha notificado que diversas son las patologías que se presentan en la población, por ejemplo, las enfermedades cardiovasculares que influyen en la presión arterial y se asocian a causas como: el tabaquismo, el consumo de alimentos ricos en grasas y la obesidad; esta última considerada como enfermedad y epidemia a la vez. La hipertensión arterial se relaciona según la OMS (2019) con la afroascendencia, la obesidad, la ansiedad y la herencia. Sea cual sea su causa, hoy día cobran miles de vidas por año.

En ocasiones, la hipertensión se agrava porque es asintomática y su atención es esencial porque se refleja en dolor de cabeza muy intenso y repentino, dificultad para hablar y levantar un brazo o parálisis facial, mareos o vértigo, visión borrosa, dolor en el pecho o sensación de falta de aire (Ortiz et al., 2017).

El estudio es parte del proyecto de investigación: “Zona protegida, Parques La Rotonda y Las Vegas de Portoviejo", como iniciativa del Instituto Superior Tecnológico "Portoviejo", donde sin dudas, la presencia de los estudiantes del ITSUP en los parques, como promotores y educadores para la salud ha sido esencial en la revalorización por la vida y el cultivo de la conciencia en beneficio de la salud integral.

El presente artículo tiene por objetivo, sensibilizar a las personas hipertensas en el control de la presión arterial en función de que puedan lograr un estilo de vida saludable.

\section{Materiales y métodos}

La investigación se realizó en el Instituto Superior Tecnológico "Portoviejo" en la provincia de Manabí, Ecuador. El quehacer pedagógico de la educación superior ecuatoriana se centra en tres 
funciones sustantivas, entre ellas la vinculación con la sociedad. Justamente, el estudio responde a un proyecto donde se establece la relación entre la institución y la comunidad. En este orden, vale destacar que el proyecto se denominó "Zona protegida en los Parques La Rotonda y Las Vegas de Portoviejo", el que tuvo lugar entre los meses de junio y diciembre de 2019.

El estudio fue de corte transversal y tuvo en cuenta la participación de 500 personas comprendidas entre 30 y 80 años de edad; quienes dieron su consentimiento para la participación voluntaria en la investigación. Personas que se fidelizaron al proyecto, por el impacto y por la calidad del servicio prestado de manera ininterrumpida por docentes y estudiantes del área de salud del instituto.

El trabajo con los participantes de estudio, tuvo basamento en variables debidamente identificadas: edad, tiempo de presencia de la enfermedad, cuantificaciones y control de la tensión arterial, género y estilos de vida. Las técnicas utilizadas fueron: registros de control de usuario del proyecto de vinculación con la sociedad, la observación y la entrevista.

Para el abordaje teórico - metodológico del control de la presión arterial y su influencia en el estilo de vida de las personas hipertensas, se partió de la imbricación de métodos teóricos, empíricos y estadísticos. Entre ellos se emplearon el análisis - síntesis, inductivo - deductivo e histórico - lógico; lo que permitió la profundización en la teoría consultada.

Por su otra parte, se aplicó una encuesta donde se tuvieron en cuenta elementos relacionados con los controles de la presión arterial, comportamiento de las personas hipertensas y estilo de vida. Para la solución de la problemática identificada, se influyó en las necesidades sociales, la influencia del trabajo preventivo a través del proyecto de vinculación con la sociedad.

Los métodos estadísticos empleados coadyuvaron al análisis de la información obtenida, a partir del diseño de una base de datos para la realización del análisis estadístico inferencial, a partir del estudio del cálculo porcentual, empleándose el programa Microsoft Office Excel; donde se consideraron las frecuencias absolutas y relativas. 


\section{Resultados}

Desde la investigación se tuvo en cuenta la edad y el género de los usuarios. En esencia la mayor cantidad usuarios atendidos fue de 300 mujeres $(60 \%)$ de edades comprendidas en el rango 30 a 50 años y 200 hombres (40\%) de edades en el rango de 50 a 80 años. Fue relevante en la entrevista que 450 usuarios $(90 \%)$ concordaron en que su debut como hipertensos fue entre los 30 y 40 años, incluso al término de la adolescencia cerca de 90 personas $(20 \%)$.

En el intercambio con los 300 usuarios más frecuentes $(60 \%)$ se evidenció un alto nivel de ansiedad por saber la cuantificación de la presión arterial. Su constancia permitió cuantificar hasta tres veces en el día; apreciándose arraigo en la enfermedad. Los valores fueron normales en cada cuantificación por la adherencia a los fármacos que los médicos le han indicado.

Las cuantificaciones más elevadas oscilaron entre 160/109 a 179/109 mmHg en 200 usuarios, de ellos 90 hombres (45\%) y 110 mujeres (55\%), que representan los menos sistemáticos. El resto que son 300 usuarios la falta de control de la presión arterial ha sido causante de complicaciones reflejadas en el estilo de vida. Un dato importante, es que no han asistido a consultas médicas para un diagnóstico certero y la prevalencia de la hipertensión arterial, según sus testimonios sobrepasan los dos años; sin control.

En sentido general, el estilo de vida es diverso, en tanto 270 usuarios refirieron (54\%) que es aceptable, 130 que es medianamente aceptable (26\%) e inaceptable 100 usuarios (20\%).

\section{Discusión}

Es esencial el dominio de las bases teóricas relacionadas con hipertensión arterial, que es el incremento de la presión arterial y que su sintomatología aun precisa de estudios pues no tiene a manifestarse en el tiempo (Vitón \& Marcos, 2019). 
$\mathrm{Su}$ criterio se refleja a la luz de que es una patología tratable pero su descuido es mortal. Sin lugar a dudas, desdeñar las recomendaciones médicas pueden acarrear consecuencias graves e irreversibles incluso. Se asocia a enfermedades cerebrovasculares, entre otras que son evitables a través del control. En este sentido, 275 usuarios (55\%) desconocen que son hipertensos, así como las consecuencias de la falta de control de la presión arterial; en tanto el resto (45\%) conocen que son patológicos pero no las consecuencias de la falta de control de la presión arterial (Ortiz, Torres, Cordero, Lara, Torres, Procel. \& Bermúdez, V. 2017).

Los factores de riesgo asociados a hipertensión arterial y destacan que aún no se logra establecer con especificidad. Aluden en sus estudios a la herencia genética, la edad, el sexo y la etnia; planteando que se relacionan con los hábitos, el ambiente y las costumbres de las personas: obesidad, sensibilidad al sodio, consumo excesivo de alcohol, uso de anticonceptivos orales y el sedentarismo, en la investigación se concuerda con los autorees (Ortiz, Torres, Cordero, Lara, Bermúdez, 2017).

Es interesante notar que los hallazgos investigativos de los autores mencionados, refirieron que la hipertensión arterial es un factor de riesgo modificable para la presencia de enfermedades vasculares, cardiacas y renales, con importantes repercusiones en la salud a nivel internacional, donde Ecuador no escapa de la realidad actual. Sobre esta base se entiende que una persona es hipertensa cuando supera los valores normales de $\mathrm{mmHg}$ y transita por diferentes estadios.

Los 500 usuarios fueron capacitados en los valores relacionados con las cuantificaciones de la presión arterial; enfatizando en los 275 usuarios (55\%) descritos con anterioridad. El 100\% sentenció la importancia de los siguientes valores: Presión arterial normal: menos de 120/80 $\mathrm{mmHg}$, Prehipertensión: 120/80 a 139/89 mmHg, Estadio I de hipertensión: 140/90 a 159/99 mmHg, Estadio II de hipertensión: 160/109 a 179/109 mmHg y Estadio III de hipertensión: mayor de 179/109 mmHg. Es interesante notar que no todas las personas hipertensas saben que lo son.

¿Por qué es importante el control de la presión arterial? El 100\% de los usuarios asintieron que las consecuencias de la hipertensión arterial son graves y se evidencian en: accidente cerebrovascular, ataque cardíaco, insuficiencia cardíaca, enfermedad renal o muerte prematura. 
La forma de concientizar a los usuarios se logró a través de las acciones por etapas, que se caracterizaron por su dinamismo, operatividad y practicidad, el 100\% de los usuarios recibió la información siguiente de que las maneras de controlar la hipertensión arterial son: seguir las indicaciones médicas, reducir el consumo de sal, disminuir la ingesta de bebidas alcohólicas, ingerir una dieta saludable con cantidades de potasio y fibra recomendadas, beber suficiente agua, mantener un peso corporal sano, ejercitar el organismo, reducir los niveles de estrés, dejar de fumar si lo hace o consumir otras sustancias psicoactivas.

Aún predomina la aversión de algunos pacientes a la hora de acudir al médico ante los síntomas de lo que pudiera indicar una hipertensión arterial. Briones (2016) insistió en que la ejercitación física ayuda a controlar este padecimiento o evitarlo en quien aún no ha debutado como hipertenso.

Se concuerda con los estudios realizados por la OMS (2019), refiere que son concluyentes en cuanto a la sintomatología que indica una posible hipertensión arterial: cefalea intensa, pulsaciones cardiacas irregulares, dolor a nivel de tórax, sudoraciones, náuseas, vómitos, respiración dificultosa, mareos, dolor en algunas regiones del cuerpo (cuello, mandíbula, hombros), debilidad corporal, desmayo, visión dificultosa, pérdida de la orientación, habla confusa. En todos los pacientes no se revela del mismo modo, incluso hay pacientes hipertensos asintomáticos, (OMS, 2019).

El control de la presión arterial articula con un estilo de vida saludable. En este orden de análisis, una de las alternativas es lo que ya se ha planteado. No obstante, la mejor opción es autoeducarse pues, cada consejo es vano sino hay conciencia en sí mismo. Cada persona debe garantizar una vida sana y activa, gestando cambios aun pequeños en virtud de la salud del organismo.

También se incidió en que las consecuencias de la hipertensión arterial son evitables, las personas sucumben por la autocomplacencia de la afirmación más peligrosa del mundo: " $A$ mí no me va a pasar”. El repensar exige modificar patrones de conducta personal o individual, pues las decisiones que se tomen afectan al propio paciente y a la familia de este. Esta modificación exige desarraigarse preferencias y hábitos; encontrando con la guía médica alternativas de bienestar.

La cantidad y calidad del sueño, son factores de hipertensión arterial. Aseveran que la depresión y el estrés, están estrechamente relacionados con la patología, objeto de estudio de esta investigación. Sus 
hallazgos dirigieron la atención a que el personal de salud debe coadyuvar a la promoción de la salud, prevención de enfermedades y evaluación de los estilos de vida. (Hernández de la Rosa, Godoy, Romero, Gutiérrez \& Arthur, 2018, pág. 155).

Durante la ejecución de las acciones, se establecieron siete recomendaciones en función del estilo de vida en las personas hipertensas:

- Repensar en la vida, en los objetivos y en estrategias de mejoramiento.

- Elevar la autoestima e identidad.

- Autodecidir, autogestionar y aprender de manera permanente.

- Integrarse familiarmente, autocuidarse.

- Suscitar la conciencia personal y la de otros.

- Ser feliz.

- Distraerse y disfrutar el compartir con los demás.

Implica ser resiliente y encausarse hacia la conquista de un estado de ánimo optimista y de felicidad plena, rechazando la aflicción y la depresión. La práctica del ejercicio de la mente sin importar la edad y mantener la decisión de no dejarse perturbar.

En estudio sobre competencias humanas, García (2015) declaró que: el centro de la competencia es la motivación" y solo los seres humanos se motivan. Esto quiere decir, que el mejoramiento de los estilos de vida en una persona hipertensa o no, depende de la motivación que tenga hacia la vida. Aun cuando es altamente subjetiva la afirmación, una persona feliz puede evitar la manifestación o el descontrol de la hipertensión arterial.

\section{Conclusiones}

La presión arterial es una enfermedad crónica multicausal, que no se relaciona ni con la edad ni con el género; por su parte tiene su génesis en el consumo excesivo de sal y de bebidas alcohólicas, las dietas carentes de potasio y fibra, el insuficiente consumo de agua, el sobrepeso, la obesidad, el sedentarismo, el estrés, la ansiedad y el consumo de drogas legales e ilegales. 
La presión arterial ha sido estudiada por diferentes especialistas del área médica y otras, a través de la revisión bibliográfica se concluye de que a pesar de ser una patología crónica puede ser controlada y de este modo influir el estilo de vida de las personas hipertensas.

El objetivo fue cumplido, en torno a la educación de las personas hipertensas que formaron parte de la investigación; a las que se les capacitó en los buenos hábitos relacionados con la alimentación, la nutrición, el ejercicio físico, la reducción del estrés y la ansiedad; propendiendo a una comunidad poseedora de una vida saludable.

\section{Bibliografía}

Briones, E. M. (2016). Ejercicios físicos en la prevención de hipertensión arterial. Medisan, 20(1), 35 41. Recuperado de: http://scielo.sld.cu/pdf/san/v20n1/san06201.pdf

Cachimuel, E. E. (2020). Estilos de vida en pacientes hipertensos de 45 a 75 años que acuden al centro de salud Tipo C-San Rafael, Esmeraldas 2019 (Bachelor's thesis).

De la Rosa, J. M., \& Acosta Silva, M. (2017). Posibles factores de riesgo cardiovasculares en pacientes con hipertensión arterial en tres barrios de Esmeraldas, Ecuador. Revista Archivo Médico de Camagüey, 21(3), 361-369. Recuperado de: http://scielo.sld.cu/pdf/amc/v21n3/amc070317.pdf

Hernández de la Rosa, M., Godoy Quinto, J., Romero San Salvador, C. Y., Gutiérrez Gabriel, I., \& Arthur Aguirre, F. (2018). Efecto del estilo de vida en el control de pacientes con hipertensión arterial sistémica en una unidad de medicina familiar en Puebla, México. Atención Familiar, 25(4), 155-159. Recuperado de: https:/www.medigraphic.com/pdfs/atefam/af-2018/af184f.pdf

Leyton, M., Batista, M., \& Jiménez-Castuera, R. (2020). Modelo de predicción de los estilos de vida saludables a través de la teoría de la autodeterminación de estudiantes de educación física. Revista de Psicodidáctica, 25(1), 68-75. Recuperado de: https://doi.org/10.1016/j.psicod.2019.05.002

Morejón Giraldoni, A. F., Benet-Rodríguez, M., Bernal-Muñoz, J. L., Espinosa-Brito, A. D., Silva Aycaguer, L. C., \& Ordunez, P. (2020). Factores relacionados con el control de la hipertensión 
arterial en Cienfuegos. Revista Cubana de Salud Pública, 45, e1716. Recuperado de: http://www.revsaludpublica.sld.cu/index.php/spu/article/view/1716

Organización Mundial de la Salud (2019): El cuerpo y la mente saludable. Recuperado de: https://www.salud.mapfre.es/cuerpo-y-mente/habitos-saludables/la-importancia-de-cuidar-lasalud/

Ortiz, R., Torres, M., Cordero, S. P., Lara, V. A., Torres, M. S., Procel, X. V. \& Bermúdez, V. (2017). Factores de riesgo asociados a hipertensión arterial en la población rural de Quingeo Ecuador. Revista Latinoamericana de Hipertensión, 12(3), 95-103. Recuperado de: https://www.redalyc.org/pdf/1702/170252187004.pdf

Otero, J., Camacho, P. A., Gómez-Peña, L. M., Rueda-Quijano, S. M., Gómez-Cuellar, J. F., Rey, J. J, $\&$ Arcos, E. (2020). Medición, control y conocimiento de la presión arterial: Iniciativa Mayo Mes de la Medición Colombia 2017. Hipertensión y Riesgo Vascular, 37(1), 4-10. Recuperado de: https://pesquisa.bvsalud.org/portal/resource/pt/ibc-188667

Vitón, I., \& Marcos, J. (2019). Estilos de vida y presencia de hipertensión arterial en el adulto mayor del Centro de Salud Conchán, Chota, Cajamarca-2018. 